Department of Philosophy Abo

Akademi, Finland

\title{
Craig Dilworth
}

\section{PRINCIPLES, LAWS AND THEORIES}

This paper is essentially a summary of the central part of an ongoing project in which the attempt is being made to provide a comprehensive conception of modern science. A preliminary word might be said about the aim and method of the project. Its aim is not at all to be prescriptive, i.e. to suggest that modern science or any other epistemological activity ought to be pursued in the way depicted here. Nor, however, is it intended to be descriptive - to provide general truths about the nature of science. Rather, one might say that the aim of the project is to be explanatory, that is, to provide a coherent understanding of modern science in which all its major aspects have a place, and in which its basic limits become clear.

This aim in part determines the method, which will involve presenting idealized representations of various aspects of science, which are to constitute conceptual paradigms around which science takes its form. In this way the attempt is made to capture the essence of modern science, while at the same time admitting the existence of counterexamples and borderline cases with respect to the features characterized. In this regard, as will be seen below, the present method has certain similarities with the scientific method it depicts.

The notions of principles, laws and theories are here taken as constituting the basic categories in terms of which science is to be conceived. Very briefly, we may say that principles are of two sorts, which may be called metaphysical (often implicit) and scientific (often explicit), and that in both forms they underlie empirical laws and theories. Empirical laws for their part, are discovered following a methodology implied by the principles, while theories are propounded to explain the laws by 
showing explicitly how they are but manifestations of the operation of the principles in nature.

1. PRINCIPLES. Those who consider science to have a foundation usually see that foundation as lying in the empirical or experimental results of scientific activity. Here, however, the basis of science will not be seen to rest in empirical data, but in metaphysical principles. In fact, modern science as here conceived consists essentially in the application of certain particular metaphysical principles to various aspects of the real world $L$ These metaphysical principles are themselves obtained on the basis of reflection on the nature of reality, and may be variously conceived: some of them may be considered more fundamental than others, or they may be divided up in different ways, or be taken as constituting a unity and not be divided up at all ${ }^{1}$. It is not of primary importance to the present view just how this is done, but for reasons some of which will become apparent as this essay proceeds, the metaphysical principles underlying modern science have here been taken to be three in number, and, more particularly, to be the following:

A. The principle of the uniformity of nature. This principle is usually understood to mean that nature is lawful, that natural change takes place according to rules. Cast in a more definite form, the modern version of the principle says that the nature of change is not affected by mere spatial or temporal location, such that if at one place and time certain conditions were sufficient for the occurrence of a particular event (change), they would be sufficient at any place and time. In this formulation the principle may be seen as being about space and time, suggesting that both are in a sense homogeneous.

The principle of the uniformity of nature is to apply to change, which gives rise to the question of what change itself is.

1 For thoughts in a similar vein, cf. e.g. Duhem (1906), p. 335, where he says: „it would be unreasonable to work for the progress of physical theory if this theory were not the increasingly better defined and more precise reflection of a metaphysics; the belief in an order transcending physics is the sole justification of physical theory". It has been suggested in discussion that the adoption of principles in science as characterized here is a 'bootstrapping' procedure. Though the present account and that of those who speak of bootstrapping may be motivated by similar epistemological considerations, the bootstrapping approach is quite alien to the present one due to its logicism. For an extended argument against logicism in the philosophy of science, see Dilworth (1986); for 'bootstrapping' see e.g. Earman and Glymour (1988).

2 F. S. C. Northrop, for example, who also sees science as resting on principles of the sort envisaged here, divides them up differently: cf. Northrop (1931), pp. 24-25.

B. The principle of substance. This principle is to the effect that all change consists in the transformation of an eternally existing substance. An important implication of the principle is that substance, so conceived, can be neither created nor destroyed. Given the principle, substance is paradigmatically conceived to be material and to occupy all or a part of space, and its transformation to consist in the motion of its parts. It should also be noted, however, that motion (change) itself may be considered a substance, i.e. something which, while taking different 
forms, exists perpetually ${ }^{3}$.

Assuming that change consists in the transformation of substance, it may be asked how that transformation is brought about.

C. The principle of causality. This principle states that all change is caused. It is fundamentally different from the principle of the uniformity of nature in that it does not concern lawfulness, or the following of a rule, but cause, or the production of an effect. Where the principle of the uniformity of nature implies a regularity or determinism in nature without specifying a reason for this regularity, the present principle implies that there is no change without a cause, but does not suggest that causes need be regular in their effects.

Taken together, however, these two principles do imply tha ${ }^{1}$ like causes have like effects, as well as that space and time are causally inert; and in conjunction with the principle of substance as paradigmatically conceived, that both cause and effect consist in the motion of substance.

It is important to note that the above principles are not here intended to be conceived e.g. simply as statements about the nature of reality - statements the truth of which may or may not be presupposed in the doing of science. Rather, they are more to be thought of as constituting conceptual paradigms in terms of which scientific thought is conducted. This means that they constitute ideals for the doing of science, not that they are slavishly followed in every activity deserving to be called scientific. They have greater difficulty finding application in the social sciences than in the natural sciences, and even in the natural sciences the conceptual reforms demanded by 20th century physics lead to their being seriously questioned. But what must be emphasized here is the lack of clear-cut positive alternatives to take their place - alternatives which are of equal simplicity and apparent generality - either in the social sciences or in modern physics. Thus, just as important as the question of the extent to which these principles have been accepted or rejected through the history of modern science, is the idea that they provide the 77.

${ }^{3}$ The idea of the substantiality of motion is discussed in some detail in Capek (1961), pp. $71-$ 
fundamental categories of scientific thought ${ }^{4}$. In this way they constitute the core of the conceptual framework in the context of which science is pursued, so that even when they are not followed - and counterexamples abound - what is followed is framed in terms of them.

As the attempt will be made to show now, these three metaphysical principles all have counterparts in modern science as explicit scientific principles, whose form is determined in part by the nature of the subject matter being investigated, and in part by the stage of the investigation.

Ai. The principle of the uniformity of nature is manifest most directly in modern science in terms of principles of spatial and temporal invariance (and covariance). Understood as being about space and time, the principle of the uniformity of nature is itself the most general scientific principle of this kind 5 .

Within physics, the principle becomes refined in various ways. Thus we have the invariance or covariance (invariance of form) of particular physical laws with respect to different states of motion, for example the covariance principles of special and general relativity.

Principles of invariance and covariance are indifference principles, i. e. of the form: 'It does not matter whether...'. Another example falling under the general principle of the uniformity of nature is the principle of the indifference of spatial orientation. This, like the principle of relativity, is a symmetry principle, and has the implication that space is isotropic.

Another scientific uniformity principle which might be mentioned here is the principle of the relativity of magnitude, or the principle of scalar indifference, which has implications concerning e.g. efforts to delineate fundamental particles and smallest quanta of action.

$\mathrm{Bi}$. The principle of substance is most clearly manif est in modem science as the fundamental principle of chemistry, namely, the principle of the conservation of matter, according to which in chemical processes matter never changes in quantity but only in form. In physics the corresponding principle is that of the conservation of mass, which has been refined to become the principle of the conservation of energy

\footnotetext{
${ }^{4}$ The importance of categories with regard to scientific thought is also of concern to Einstein where, with regard to realism, he says: „We are here concerned with 'categories' or schemes of thought, the selection of which is, in principle, entirely open to us and whose qualification can only be judged by the degree to which its use contributes to making the totality of the contents of consciousness 'intelligible' "' (1949, p. 673).

Wigner expresses the principle of the uniformity of nature thus: ,given the same essential initial conditions, the result will be the same no matter where and when we realize these"; or, ,in the language of initial conditions, as the statement that the absolute position and the absolute time are never essential initial conditions". This ,is the first and perhaps the most important theorem [sic] of invariance in physics. If it were not for it, it might have been impossible for us to discover laws of nature".

„The above invariance is called in modern mathematical parlance invariance with respect to displacement in time and space" (1967, p. 4). Note the lack of reference to causal notions.
} 
(mass being essentially equated with energy) ${ }^{6}$.

Here we see how the one metaphysical principle can concern different aspects of reality (matter vs. mass or energy) when employed with respect to different disciplines, and how when employed within one discipline it can evolve (conservation of mass to conservation of energy) as the aspect it concernes is reconceptualized.

Other conservation principles may also be mentioned, such as conservation of linear and angular momentum (which assume motion to be a substance), parity (in all but some weak interactions), strangeness (in strong but not weak interactions), charge, lepton number and baryon number. While the very idea of framing various conservation principles stems from the principle of substance, those mentioned here involve other of the basic principles as well, i.e. they have spatial, temporal and/or causal aspects.

Ci. The metaphysical principle of causality is perhaps most clearly ma nifest in science as the principle of inertia: that a body will continue in its state of rest or uniform motion in a straight line unless acted upon by a force. Here the notion of force is the physical specification of the metaphysical notion of cause ${ }^{7}$; and change consists in change of state (rather than change of position) due to the presupposition of the substantiality of motion. Thus the principle of inertia implies that there are no uncaused changes of state.

It may be noted that not all principles in science are called by that name, and some are more often referred to as laws, for example, Newton's three laws of motion and the laws of thermodynamics.

The metaphysical principles mentioned above, as well as their counterparts in science, concern four main notions: space, time, substance and causality - the first principle having to do with space and time, the second with substance, and the third with causality. It may be suggested that these four notions are in fact the most fundamental notions of natural science, and that all its principles are framed in terms of some combination of them.

Even more basic than these, however, are the notions of constancy and change; and we may say that all principles of natural science have to do with what changes and what does not ${ }^{8}$.

${ }^{6}$ Campbell recognizes the relevance of distinguishing between laws and principles in this context, where he says: „The 'laws' of a conservative system are not really laws, but for the present they may pass as such" (1920, p. 133n.).

We note here also that, as has been pointed out in discussion by Yves Gingras and Franęoise Balibar, in physics conservation and invariance are closely related notions. Conservation always implies an invariance of something (the substance of the discipline); and invariance always implies conservation (of form, if not of substance). For present purposes, however, in keeping with the practice of physicists, it is nevertheless important to maintain, a distinction between the two notions.

${ }^{7}$ Much attention is devoted to this point in Whewell (1858-1860), vol. 1, pp. 172ff., 236ff.

${ }^{8}$ On this point, cf. e.g. Harre (1970), pp. 249—250, where it is suggested that „The changing is explained in terms of the unchanging... Chemical change is explained by unchanging atoms, atomic 
There are at least five different ways in which principles can be seen to function with respect to science. The first way is that they set limits on what can be conceived as possible within the enterprise or its sub-disciplines. It is of interest to compare this with the 'possible worlds semantics' currently attracting attention in linguistic philosophy. The possible worlds delinated by the principles of science are not to be identified with logically possible worlds, but with physically or scientifically possible worlds. Scientific principles are much more restrictive regarding what they admit than is logic 9 .

Through delimiting what is to be conceived as possible, principles determine the scientist's way of thinking, that is, they provide the structure of scientific rationality, which is their second function. Rationality in general is not equivalent to logic, as has been supposed not only by those in the logicist tradition but even by some of their detractors ${ }^{10}$; nor is scientific rationality equivalent to logic. It is principles that determine the point beyond which it no longer makes sense to ask for a reason; and in science this point consists in the indication of how what is to be explained is but a manifestation of the principles on which the enterprise is based.

Thus, as far as science is concerned, principles determine both what is to be considered possible and what is to be considered rational, thereby as much as fulfilling their third function, which is to set guidelines for the actual doing of science. In this context two types of principles may be distinguished. There are on the one hand, spatio-temporal, substantial and causal principles, as given above, all of which may broadly be termed ontological; and on the other hand there are methodological principles, which may be considered as rules for the doing of research. On the present view, methodological principles stem from ontological principles. This view makes it idle simply to advance methodological principles without giving them an ontological grounding, as has frequently been done throughout the history of reflection on science ${ }^{11 .}$

Methodological principles guide research throughout the whole of a branch of science, whether that research be emprical or theoretical. Thus theoreticians, for their part, are led to construct specific causally efficacious ontologies in which the ontological principles are strictly adhered to, while experimentalists create conditions in the laboratory in which the ontological principles can be unequivocably manifest $\mathrm{t}^{12}$.

A fourth way in which principles can function with respect to science is as definitions of its basic concepts, thereby delimiting the nature either of the enterprise as a whole, or of its various sub-disciplines. A change in a discipline's principles may thus be seen as indicating a change in the nature of the discipline; and a sufficiently

change by unchanging fundamental particles".

${ }^{9}$ Concerning the divorce of science from logic in this context, cf. Whewell (1858-1860), vol. 4, p. 342: „I will not pretend to say that this kind of necessity [logical necessityl is represented by any of those Fundamental Ideas which are the basis of science".

${ }^{10} \mathrm{Paul}$ Feyerabend, for example, in attacking what he believes to be the 'rationalism' of Popper and Lakatos, does not offend rationalism at all, but Popper's and Lakatos' particular brand of logicism: cf. Feyerabend (1975) and Dilworth (1986). 
drastic change can lead one to question whether it is still the same discipline. Together with the third way discussed above, this may provide a profitable means of understanding Thomas Kuhn's notions of paradigm and scientific revolution. Here the paradigm around which a discipline is constructed, and in terms of which its basic notions are defined, consists of nothing other than its fundamental principles; and scientific revolution consists in a change in or of those principles.

11 To mention but two examples, Newton's Rules of Reasoning in Philosophy (1687, pp. 398400), and Popper's Three Requirements for the Growth of Knowledge (1962, pp. 240 -248).

12 The methodological implications of ontological principles will also undoubtedly have sociological repercussions. Given some of the considerations to be presented below, one can for example understand that the quest to discover ever more empirical laws will necessitate the use of sophisticated apparatuses which will require funding for their construction and teamwork for their operation, while the provision of theories will not demand such expenditures and can be of a more solitary nature. And one can appreciate that as regards both empirical and theoretical pursuits the universality of the subject matter of science will allow scientists to constitute an international community whose sub-parts can in principle work geographically independently. This is a theme which can of course be developed further.

The fifth role that may be assigned to principles is that of being statements about the nature of reality. But even in this guise, as has been pointed out by Poincare and others, they are in no straightforward v/ay either verifiable or falsifiable. They are of course too general to be verified; and while empirical research may lead to a principle's being emended, or even discarded, one can never be sure that appears to be a falsification is not due merely to the principle's being improperly applied ${ }^{11}$.

In that principles pervade the whole of a discipline, anyone working within the discipline is doing so in accordance with principles. Even if it were the case that he consciously denied the viability of a particular principle or principles with regard to his research, just by doing so he would be adopting their denials as principles. Not to adopt a principle is to adopt a different principle; and in any discipline they are something with regard to which one has a position, whether he be aware of it or not.

2. LAWS. We have seen above how the notions of space, time, substance and causality can be expressed in terms of metaphysical principles which have explicit counterparts in the natural sciences. Now each of the metaphysical principles can be seen as expressing only certain of these four notions, so that we could say that the principle of the uniformity of nature for example has really only to do with space and time. Explicit scientific principles, on the other hand, while each perhaps emphasizes certain of these notions, often involve a combination of them, such as in the case of the principle of inertia, which primarily concerns the notion of cause (force), but also involves e.g. the notion of substance, in that it presupposes the substantiality of motion.

It should perhaps be emphasized that these metaphysical principles themselves

13 Cf. Durhem (1906), p. 214: „Thus many a principle of mechanics has a form such that it is absurd to ask one's self: 'Is this principle in agreement with experiment or not?' This strange character is not peculiar to the principles of mechanics; It also marks certain fundamental hypotheses of our physical or chemical theories". 
determine a way of thinking (the second function of principles mentioned above), or what some might term a 'hermeneutic context', which can make itself felt in scientific methodology without ever receiving a corresponding explicit scientific formulation. This is so as regards the (ontological) principle of the uniformity of nature which, together with its metaphysical counterpart concerning reason, the principle of induction, constitute perhaps the most fundamental principles of empirical scientific investigation.

The principle of the uniformity of nature states that if at one place and time certain conditions were sufficient for the occurrence of a particular event (change), they would be sufficient at any place and time. The principle of induction, on the other hand, is usually understood to state something to the effect that if all experienced cases of one particular kind of state of affairs have been accompanied by a particular other kind of state of affairs, we may infer that this will also be so in all unexperienced cases.

Taken together, these two principles have an extremely important prescriptive implication for the methodology of empirical science, namely: acquaint yourself with the kinds of states of affairs which can in principle, and more particularly in practice, exist at different points in space and time, and consider the nature of those hitherto unknown changes which accompany them ${ }^{12}$. In other words: try to discover natural laws.

The repercussions of this prescription are manifold. For one thing, it directs empirical investigation towards states of affairs which can in essence be replicated. Also, it implies an active rather than passive investigative procedure which involves controlling the conditions under which the investigation is being performed. And it demands a means of establishing objectivity in the determination of whether two spatially or temporally distinct states of affairs are sufficiently similar to be expected to be accompanied by the same sorts of changes.

The first step in the meeting of these requirements consists in the provision of a standard, i.e. something which can be used to obtain objective information concerning spatially and temporally different situations with respect to at least one of their common properties. Not only need the standard afford a means of comparing a property's changing with its not changing, but also its different ways of changing. This creates awkwardness in that the standard itself is not allowed to change. Eut it should be possible for the standard to be replicated, whereby different configurations of its replications can be employed in objectively comparing changes in a property. Such a standard is nothing other than a unit of measurement; and its replications are measuring instruments.

Measuring instruments are not only necessary for the objective comparison of changes, but for ensuring that, with respect to the properties susceptible of measurement (parameters), the conditions from which the changes emanate are

${ }^{12}$ In this regard, cf. Campbell (1920), pp. 69-71, 111-112, and 87: ,we accomplish the apparently miraculous feat of reducing a chaotic world to order, because we carefully confine our attention and c-ur efforts only to those portions which we find can be ordered". 
determinate and themselves replicable.

The measuring instruments at one's disposal completely determine the paradigmatic kind of scientific knowledge one can obtain about the world, which is knowledge of certain laws of nature. In the simplest case the empiri cal scientist creates a situation in wchich all causally relevant parameters (forces) but one are eliminated or held constant, while the one is systematically varied; and the result of this variation as manifest in the change of another parameter is measured and recorded. This is then done successively with all of the relevant parameters. If these various operations have been properly performed, and a regular relation expressible in the form of an equation (or group of equations) is found to obtain among the parameters, the equation is taken to depict a natural law (in accordance with the principle of the uniformity of nature); and repetitions of such a procedure are made only to ensure that the operations were in fact properly performed. Through the discovery of such laws, the empirical scientist is able to learn some of the unchanging rules according to which natural change takes place.

Natural laws as expressed in science are thus not e.g. universal generalizations (wtih definite truth-values) in which entities everwhere are claimed necessarily to have particular properties if they have certain other properties. Rather, they are equations relating measurable parameters which, due to the principle of the uniformity of nature, provide information about a world which in a particular sense is abstracted from spatial and temporal considerations. Thus the expressions of laws take a mathematical (quantitative) form, and concern more particularities than generalities, albeit particularities which are highly interconnected. While they are invariably approximate (due in part to the nature of measurement), they are not conjectural, but on the contrary express the facts of science. These facts are usually manifest most clearly in highly artificial or constructed situations, and even when not, their scientific expressions are directly applicable only to ideal states of affairs, which is to say that scientific laws are themselves idealizational. Such laws are discovered; and their proper expression provides scientific knowledge.

While the principle of the uniformity of nature pervades the whole of empirical science, the principle of substance also plays an important role in this context. This principle further delineates the conditions under which laws may be discovered by requiring that no matter how the properties of a substance may change in the performance of an experiment, the substance itself must be the self-same throughout the process. This implies that the quantity of the substance is not to change, and constitutes a precondition for linking theories to laws, as will be seen below.

In physics, for example, the principle of substance is today most clearly manifest in the form of the conservation of energy: energy may take many forms, but it persists and its quantity remains unaltered whatever qualitative changes it may undergo. Other things being equal, a change in the quantity of force exerted in a particular situation will mean a change in the quantity of energy. By excluding extraneous forces, the experimental physicist tries to create a situation in which energy is conserved, i.e. to isolate a system, so that the energy change manifest in the variation in the independent parameter can equal that manifest in the dependent one. Should these two values turn out not to be equal, the principle of the 
conservation of energy suggests that all relevant causal parameters have not been excluded, an thereby affords a check on whether the experiment has been properly performed.

While principles determine the method of empirical enquiry, they need not determine the results, which are dependent on nature. On the basis of the principle of the uniformity of nature, these results are taken to be the manifestation of natural laws; and once it has been determined that the results have been properly obtained, the expressions of these laws are retained, giving science an accumulative aspect.

But the whole enterprise presumes that the results one obtains are the manifestation of the operation of the principles in nature, and it remains for the scientist to show how they are so. More specifically, his task in this context is to show how, assuming the continuing existence of the subject matter of the discipline, the empirical results obtained are in accordance with some particular refined form of the principle of causality. To do this is to explain the laws, which is the purpose of scientific theory.

3. THEORIES. That the same experimental results are obtained under the same conditions requires no explanation; consequently, as long as this is the case, what has to be explained is why the results obtained are the particular ones that they are. The task of scientific theory thus rests in indicating the nature of the causal relation between the conditions and the results, and this in such away as in keeping with there being no change in the quantity of the relevant substance. The causal relation is paradigmatically conceived to consist in the motion of the substance in such a way that cause and effect are temporally and spatially adjacent (the principle of locality). And the substance itself is conceived to occupy either a part or all of space (cf. atomistic vs. field theories).

Now the empirical law requiring explanation depicts a state of affairs in which such a causal relation is not evident - it might be, for example, a situation which apparently involves no motion. So the theory must refer to an aspect of the situation which is not directly manifest - which is presently unobserved - in order to provide its explanation. Thus theories, unlike the expressions of empirical laws, are by their very natures hypothetical when first put forward. They state that if the world actually has such-and-such a particular nature, then the relevant laws follow from the principles as a matter of course.

So theories concern both what is directly manifest and what is not. The former, which may be termed the nominal aspect of a theory's subject, is what the theory is intended to explain; and the latter, the real aspect of its subject, is what it represents as existing, in order to provide the explanation. But both are aspects of the same subject, which is the substance of interest to the particular discipline in question.

The general nature of the substance of interest to the discipline depends on the form of the principle of substance adopted in the discipline. For example, in chemistry the substance is matter, and in physics, energy. The particular nature of the substance, on the other hand, is depicted by theories. Thus there may be two theories within one discipline, one of which claims the substance to be discrete (an atomistic theory), while the other maintains that it is continuous (a field theory). 
Among other things, we see here how the particular form of the principle of substance that is adopted sets constraints on what can count as an acceptable explanation within the discipline, while still leaving room for the operation of the theoretician's imagination within those constraints.

In that a theory attempts to depict the particular nature of the discipline's substance, i.e. attempts to specify the form of what the discipline takes as always existing, we may say that it constitutes an ontology. This implies the persistence of certain properties, viz., those of the entities in the ontology, which is of relevance to the existence of natural kinds and to the fact that certain well defined states of affairs actually can be replicated. More important here, however, is that the causal relation the theory depicts is to be manifest as a relation between elements in the ontology. In other words, we may say that in order for a scientific theory to provide an explanation of empirical laws, its ontology must include causal mechanisms.

Now these mechanisms can take many and varied forms, and need not be of the archetypical sort where one physical object comes in contact with another. Limits are set on the form they can assume, however, by the particular causal principles adopted in the discipline. But to this it may be added that, quite generally in science, the conception of cause in which cause and effect are either spatially or temporally separated is adopted only as a last resort; and that, other things being equal, explanations which rely on this conception are generally not considered to be as intellectually satisfying as those which do not.

In the above way then a theory constitutes a model intended to explain what is overt in terms of what is not. Such a model is not to be confused with the state of affairs from which it may ultimately be drawn, which we here term its source-analogue, and which is often a situation the theoretician might come across in his everyday life. Thus, for example, we distinguish between the game of billiards, which may be considered to be the source in analogy with which the kinetic theory of gases is constructed, and the kinetic theory itself, or the theoretical model constituting its core.

The principles of a discipline delimit both the experimentalist's way of approaching empirical reality, as well as the theoretician's way of depicting theoretical reality. However, where the empirical researcher is free to deal with any property he can measure, the theoretician is constrained to depict only properties as can be conceptually constructed from the principles. More particularly, his task is to shpw how the changes which accompany the variation of certain empirical parameters (e.g. temperature) are really causally produced by the action of mechanisms (e.g. molecular motion), all of whose properties find expression in the principles.

But just as the experimentalist in physics is constrained by the principle of substance to create states of affairs in which energy is conserved, i.e. to construct systems, the theoretician's models must also depict systems. This is a necessary first step in the application of the theory to empirical reality, though it is also an integral part of the construction of the theory itself. The next step is to express the model in a form which makes it easily comparable with the formulation of the laws obtained form the manipulation of the empirical 
system. Empirical laws are expressed as equations between measurable parameters; theories may also be expressed as equations, though it is to be expected that some of the properties represented in such equations, while quantifiable, may not be measurable. These equations may be considered the expressions of theoretical laws. With respect to the real aspect of the theory's subject - what the theory directly represents - their task is to depict the particular form to be assumed by the principle of causality (e.g. Newton's laws of motion).

The application of the theory then consists in the construction of a particular mapping operation from parameters in the empirical laws to variables in the theoretical laws. This is to be done in such a way that all empirical parameters find an expression in substantial and/or causal notions (cf. e.g. the expression of temperature as the mean kinetic energy of molecules). And it is in this way that scientific theories function to explain empirical laws. An earlier version of this paper was presented at the Universite

du Quebec a Montreal, Queens College of the City University of New York, New York University and the Boston Colloquium for the Philosophy of Science. For helpful comments on these occasions, the author would like to thank Yves Gingras, Alberto Cordero, Peter Manicas, William Ruddick, Robert S. Cohen, Robert Ackermann, Franęoise Balibar and the other participants in the discussions.

\section{REFERENCES}

Campbell, N. R. (1920), Physics: The Elements, Cambridge: Cambridge University Press, 1920; reissued as Foundations of Science, New York: Dover Publications, 1957.

Capek, M. (1961), The Philosophical Impact of Contemporary Physics, Princeton: D. Van Nostrand.

Dilworth, C. (1986), Scientific Progress, 2nd edition, Dordrecht: D. Reidel.

Durhem, P. (1906), The Aim and Structure of Physical Theory, p. P. Wiener (tr.),

New York: Atheneum, 1981.

Earman, J. and Glymour, C. (1988), Discussion: What Revisions Does Bootstrap Testing Need? A Reply, Philosophy of Science, 55: 260-264.

Einstein, A. (1949), „Reply to Criticisms”, in P. A. Schilpp (ed.), Albert Einstein: Philosopher Scientist: 665-688, Evanston, Illinois: The Library of Living Philosophers.

Feyerabend, P. (1975), Against Method, London: Verso, 1978.

Harre, R. (1970), The Principles of Scientific Thinking, Chicago: University of Chicago Press.

Newton, I. (1687), Mathematical Principles of Natural Philosophy, A. Motte (tr.), Berkeley: University of California Press, 1934.

Northrop, F. S. C. (1931), Science and First Principles, Cambridge: Cambridge University Press. Popper, K. R. (1962), Conjectures and Refutations, New York: Harper \& Row, 1963. Whewell, W. (1958 - 1960), The Philosophy of the Inductive Sciences, London:

John W. Parker and Son.

Wigner, E. P. (1967), Symmetries and Reflections, Bloomington: Indiana University Press. 